\title{
REABERTURA DO PILORO GASTRODUODENAL APÓS SUA CERCLAGEM, EM RATOS
}

\author{
REOPENING OF PYLORUS AFTER ITS CLOSURE, IN RATS
}

\author{
Jorge Miguel Schettino César'; Andy Petroianu, TCBC-MG; \\ Agostinho Pinto Gouvêa ${ }^{3}$; Dierre Roberto Alvin ${ }^{4}$
}

\begin{abstract}
RESUMO: Objetivos: A lesão duodenal é um evento pouco freqüente, que incide em $3 \%$ a $12 \%$ dos pacientes com trauma de abdome. A sutura dessa ferida e a drenagem adequada da região é o tratamento mais utilizado nas lesões menores. Entretanto, as feridas de maior dimensão continuam sendo um desafio para a escolha do melhor tratamento. O fechamento do piloro e o desvio do trânsito digestório por meio de anastomose gastrojejunal é a conduta mais freqüente nessas situações. Os objetivos deste estudo foram verificar se há diferença entre o tempo de reabertura pilórica após sua oclusão com diferentes fios e se a vagotomia influencia nas alterações tissulares locais. Método: Foram estudados 30 ratos, submetidos à cerclagem do piloro gastroduodenal e derivação gastrojejunal. Os animais foram divididos em três grupos $(n=10)$, de acordo com o tipo de fio utilizado no fechamento pilórico: categute simples, ácido poliglicólico e polipropileno. Metade dos animais de cada grupo $(\mathrm{n}=5)$ foram também submetidos a vagotomia troncular. O estudo pós-operatório consistiu de radiografia abdominal após injeção intragástrica de contraste baritado, semanalmente até a constatação de trânsito gastroduodenal. Em seguida, as regiões pilórica e da anastomose gastrojejunal foram retiradas para análise histológica. A comparação entre os grupos foi feita pelo teste de Kaplan-Meier. Resultados: O fio de polipropileno manteve o piloro fechado por mais tempo $(36,3 \pm 11,6$ dias $)$ em relação aos demais fios $(p<0,05)$, não havendo diferença entre os fios de ácido poliglicólico $(25,8 \pm 14,2$ dias $)$ e categute simples ( $18,7 \pm 10,2$ dias). A vagotomia não influenciou no tempo de reabertura pilórica, mas acompanhou-se de menor reação inflamatória gástrica. Conclusões: O fio inabsorvível foi o mais adequado para a exclusão do trânsito pilórico e a vagotomia não influenciou no tempo de reabertura pilórica, mas reduziu a intensidade da gastrite pós-operatória (Rev. Col. Bras. Cir. 2005; 32(5): 328-331).
\end{abstract}

Descritores: Duodeno; Piloro; Vagotomia; Radiologia; Ratos.

\section{INTRODUÇÃO}

Lesão de duodeno pode estar presente em $3 \%$ a $12 \%$ dos pacientes com trauma abdominal ${ }^{1-4}$. Essa incidência corresponde a $10 \%$ dos ferimentos do sistema digestório e constitui um dos grandes desafios da traumatologia ${ }^{5}$. Na Primeira Grande Guerra, foram registrados 10 casos de trauma duodenal, perfazendo $6 \%$ de todas as feridas de intestino delgado, com uma mortalidade de $80 \% .^{2}$ Desde então, o seu número vem aumentando, principalmente em decorrência de acidentes automobilísticos, além de agressões por armas branca e de fogo $\mathrm{o}^{6-8}$. Essa lesão pode também ocorrer como complicação iatrogênica, em $1 \%$ a $7 \%$ dos procedimentos endoscópicos sobre o duodeno e a via biliar ${ }^{1}$, bem como no tratamento cirúrgico da úlcera péptica gastroduodenal ${ }^{3}$. Fístula e infecção são as principais complicações intra-abdominais das lesões de duodeno e ocorrem em $2 \%$ a $14 \%$ dos $\operatorname{casos}^{4,9}$.

Não há consenso em relação ao tratamento mais adequado para lesões duodenais ${ }^{5,10,11}$. A maior parte dos autores concorda que a melhor operação para as feridas simples é a sutura em dois planos e a drenagem adequada da região. Essa conduta é suficiente em $75 \%$ a $85 \%$ de todas lesões restritas ao duodeno ${ }^{6,10-13}$. Já nas lesões complexas e mais extensas, prefere-se a duodenectomia total acompanhada de pancreatectomia cefálica ${ }^{8,14,15}$.

Entretanto, a tática operatória para tratar lesão duodenal de nível intermediário continua controversa ${ }^{11}$. O fechamento pilórico é utilizado clinicamente com sucesso ${ }^{4,9}$. Algumas questões permanecem sem resposta, em relação à cirurgia de exclusão pilórica e derivação gastrojejunal, entre as quais destacam-se a opção pelo fio de sutura, de acordo com a sua eficácia em manter o piloro fechado durante o período necessário para sarar a lesão duodenal. É importante ainda saber a influência da vagotomia nos resultados dessa operação.

\section{MÉTODO}

Este trabalho seguiu as normas do Colégio Brasileiro de Experimentação Animal e foi aprovado pela Comissão de Ética do Departamento de Cirurgia da Faculdade de Medicina da UFMG.

Foram estudados trinta ratos da raça Holtzman, que permaneceram confinados em gaiolas apropriadas com cinco animais por gaiola ${ }^{16} \mathrm{e}$ livre acesso à água e ração.

1. Cirurgião Geral; Cirurgião Torácico; Mestre em Cirurgia pela Faculdade de Medicina da UFMG.

2. Professor Titular do Departamento de Cirurgia da Faculdade de Medicina da UFMG; Livre-Docente da Escola Paulista de Medicina - UNIFESP; Livre-Docente da Faculdade de Medicina de Ribeirão Preto - USP; Doutor em Fisiologia e Farmacologia - ICB-UFMG; Pesquisador 1A do CNPq.

3. Médico Patologista do Hospital das Clínicas da UFMG; Doutor em Patologia.

4. Médico Radiologista do Hospital das Clínicas da UFMG; Membro do Colégio Brasileiro de Radiologia.

Recebido em 26/09/2005

Aceito para publicação em 30/11/2005

Conflito de interesse: nenhum

Fonte de financiamento: CNPq, FAPEMIG.

Trabalho realizado no Departamento de Cirurgia da Faculdade de Medicina da Universidade Federal de Minas Gerais - UFMG, Belo Horizonte, MG. 
Os animais foram distribuídos aleatoriamente em três grupos $(\mathrm{n}=10)$, de acordo com o material do fio 4-0 utilizado para o fechamento do piloro e confecção da anastomose gastrojejunal:

Grupo 1 - polipropileno (Prolene, Ethicon),

Grupo 2 - ácido poliglicólico (Dexon, Ethicon),

Grupo 3 - categute simples. (Ethicon).

Cada grupo foi dividido em dois subgrupos $(n=5)$ :

A - sem vagotomia troncular,

$\mathrm{B}$ - com vagotomia troncular.

As operações foram conduzidas sob anestesia com éter sulfúrico. As cirurgias foram realizadas através de laparotomia mediana.

Nos animais submetidos à vagotomia, foi realizada inicialmente a secção troncular do vago ao nível da junção esofagogástrica ${ }^{14}$. Em seguida, todos os animais foram submetidos à confecção da anastomose gastrojejunal pré-cólica isoperistáltica látero-lateral com um centímetro de comprimento e sutura contínua em plano único, utilizando o fio proposto para o grupo a que o rato pertencia. $\mathrm{O}$ piloro foi ocluído com um ponto transfixante, utilizando o mesmo fio da anastomose (Figura 1).

Os animais foram submetidos a estudos radioscópicos contrastados, sob anestesia com éter sulfúrico, a cada sete dias, até a constatação de trânsito pilórico. $\mathrm{O}$ estudo radiográfico constou de injeção de $8 \mathrm{ml}$ de sulfato de bário diluído a $50 \%$ (Bariogel®), através de cateter orogástrico 6 Fr. Observou-se, à radioscopia, o trânsito digestório por até dois minutos ${ }^{17,18}$. Em seguida, ele era registrado em radiografia.

Decorrido o período de acompanhamento, os animais foram mortos com dose excessiva de éter sulfúrico. As regiões pilórica e da anastomose foram retiradas e processadas para cortes histológicos, corados com hematoxilina e eosina. A análise histológica foi feita sem o conhecimento prévio do grupo a que a peça operatória pertencia. A intensidade do processo inflamatório foi avaliada por método semiquantitativo sob luz comum e polarizada. ${ }^{19}$

Os parâmetros estudados foram comparados por meio dos testes de Kruskal- Wallis, qui-quadrado, análise de variância e Kaplan-Meier. As comparações entre os tipos de fios com relação ao tempo de abertura do piloro, intensidade da fibrose e infiltrados mono e polimorfonucleares foram realizadas pelo teste de Kruskal-Wallis. O teste de qui-quadrado e análise de variância foram utilizados para avaliar a relação entre os tipos de fio e a presença de células gigantes, identificação microscópica do fio cirúrgico e presença de células mononucleares, polimorfonucleares e atrofia da mucosa gástrica. O teste de Kaplan-Meier também analisou a presença de diferença entre os três tipos de fios em relação ao tempo de reabertura do piloro. Considerou-se o nível de significância para diferenças superiores a 95\%. $(\mathrm{p}<0,05)$.

\section{RESULTADOS}

Os animais apresentaram boa evolução pós-operatória, sem alterações dos hábitos alimentares e de comportamento. A cavidade abdominal apresentou aderências entre a região
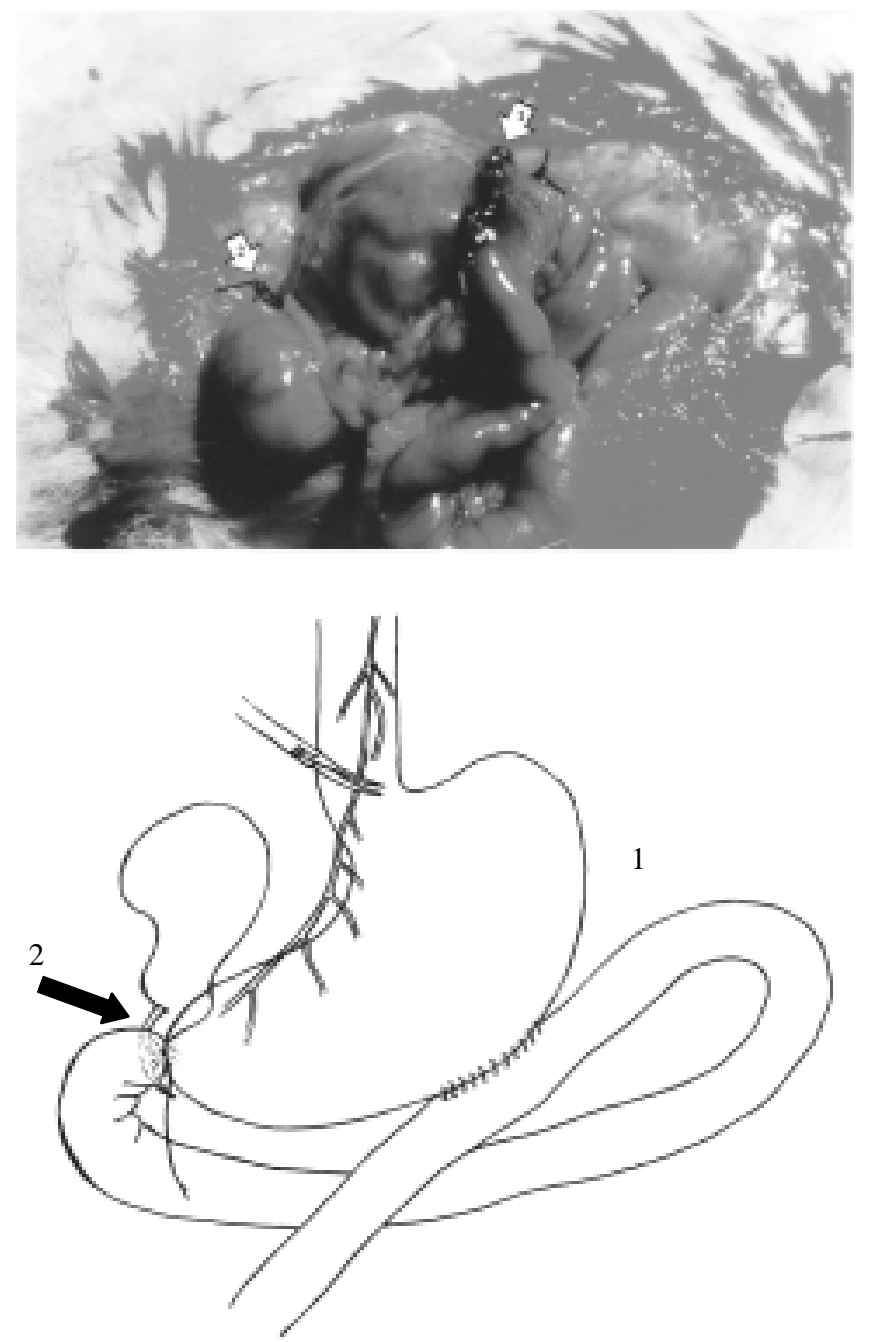

Figura 1- Aspecto cirúrgico final, mostrando a anastomose gastrojejunal (seta 1) e ofechamento pilórico (seta 2).

pilórica e a face ínfero-medial do fígado, e entre a anastomose gastrojejunal, o omento maior e o cólon transverso.

De acordo com o método do presente trabalho, o fio inabsorvível de polipropileno manteve o piloro fechado por tempo superior ao dos fios absorvível de categute simples (p $=0,003)$ e o fio hidrolisável de ácido poliglicólico $(\mathrm{p}=0,047)$. Não houve diferença entre os fios de categute simples e de ácido poliglicólico, em relação ao tempo de reabertura pilórica $(\mathrm{p}=0,113)$ (Tabela 1$)$.

Houve a probabilidade de $40 \%$ e $60 \%$ de os animais dos grupos de ácido poliglicólico e categute simples, respectivamente, estarem com o piloro aberto após 11 a 20 dias da cerclagem, contra apenas $10 \%$ dos ratos do grupo com fio de polipropileno. O piloro permaneceu fechado após 30 dias em cinco animais do Grupo 1, com fio de polipropileno, um no Grupo 2, com fio de ácido poliglicólico, e nenhum no Grupo 3, com fio de categute simples.

Os fios foram vistos ao estudo histológico no Grupo 2, com ácido poliglicólico e Grupo 3, com categute simples, nas proporções de $80 \%$ e $50 \%$, respectivamente. Ao exame com luz polarizada encontraram-se mais fios no Grupo 2, com ácido poliglicólico (90\%), seguido pelo Grupo 3, com categute simples (50\%) e Grupo 1, com polipropileno (20\%), $(\mathrm{p}=0,007)$. 
Tabela 1 - Análise comparativa dos tempos de reabertura do piloro (dias), utilizando os fios de polipropileno, de ácido poliglicólico e de categute simples.

\begin{tabular}{lccc}
\hline Fio & \multicolumn{1}{c}{ Média \pm DPM Vagotomia } & Média \pm DPM \\
\hline \multirow{2}{*}{ Polipropileno } & $36,3 \pm 11,6^{*+}$ & Sem vagotomia & $38,4 \pm 9,4$ \\
& & Com vagotomia & $34,2 \pm 12,1$ \\
Ácido poliglicólico & $25,8 \pm 14,2^{*}$ & Sem vagotomia & $28,4 \pm 15,3$ \\
& & Com vagotomia & $20,8 \pm 10$ \\
Categute simples & $18,7 \pm 10,2^{+}$ & Sem vagotomia & $24,4 \pm 13,4$ \\
& Com vagotomia & $18,4 \pm 6,9$ \\
\hline
\end{tabular}

* Diferença entre polipropileno e ácido poliglicólico com $p=0,047$.

+ Diferença entre polipropileno e categute simples com $p=0,003$.

DPM - Desvio padrão da média.

A intensidade da fibrose na parede da região pilórica foi maior nos ratos operados com fio (hidrolisável) de ácido poliglicólico, em relação aos fios de categute simples (absorvível) e de polipropileno (inabsorvível) $(\mathrm{p}=0,011)$. Também a intensidade do infiltrado inflamatório na parede da região pilórica foi maior nos ratos operados com fio (hidrolisável) de ácido poliglicólico, em relação aos fios de categute simples (absorvível) e de polipropileno (inabsorvível) $(\mathrm{p}<0,05)$. Na região pilórica, a presença de células gigantes foi maior no Grupo 2, seguida pelo Grupo 3 e Grupo1.

A vagotomia não influenciou no tempo de reabertura do piloro. Entretanto, os animais vagotomizados tiveram menor reação inflamatória na mucosa gástrica da região pilórica, principalmente no grupo com fio de polipropileno. Entre os animais não vagotomizados houve maior reação inflamatória na parede da região pilórica principalmente naqueles operados com fio de ácido poliglicólico. Não foram encontradas outras diferenças em relação aos achados histológicos entre os dois subgrupos de cada grupo estudado.

\section{DISCUSSÃO}

Na literatura, há controvérsias sobre o tratamento do trauma duodenal com fechamento pilórico e desvio de trânsito digestório ${ }^{5-7,10,20,21}$. O local onde é feita a anastomose ${ }^{22}$ pode alterar a dinâmica do esvaziamento gástrico. Anastomoses próximas ao fundo do estômago dificultam o esvaziamento gástrico, aumentando a estase, quando houver oclusão piloroduodenal.

Contrariamente ao relato de outros autores, ${ }^{10,21}$ na presente pesquisa não se encontrou diferença entre os fios de ácido poliglicólico e categute simples, apesar de a média de permanência do fio de ácido poliglicólico ter sido maior. É possível que a diferença entre esses fios seja significativa com um número maior de animais. De qualquer maneira, com ambos os fios, o piloro manteve-se fechado durante períodos inferiores ao tempo de oclusão com o fio de polipropileno. Portanto, é preferível, conforme já ressaltado previamente ${ }^{10}, \mathrm{o}$ uso de fio inabsorvível, que proporciona maior segurança ao tratamento da lesão duodenal, principalmente quando ocorrer fístula lateral ${ }^{10,20}$.

Os mecanismos de desaparecimento dos fios cirúrgicos e recanalização pilórica ainda não foram esclarecidos. Entretanto, os achados macro e microscópicos mostraram que o piloro reabre mesmo quando ocluído por fio inabsorvível. O local em que o piloro é suturado torna-se espessado, podendo englobar o fio em um processo de reação a corpo estranho, reduzindo sua resistência e iniciando a recanalização pilórica do centro da obstrução para a periferia, conforme foi observado em alguns animais deste trabalho e é relatado na literatura ${ }^{19}$. É possível também que o fio migre até a luz do duodeno e seja eliminado pelo trânsito digestório junto com as fezes. Neste estudo, não foi encontrado fio na luz gástrica ou duodenal.

As alterações da motilidade gástrica após vagotomia, descritas em outros estudos ${ }^{18}$, não foram pesquisadas no presente trabalho, que visou apenas ao estudo da repercussão da denervação parassimpática na mucosa gastroduodenal perianastomótica.

Úlcera anastomótica não foi evidenciada no presente estudo, corroborando publicações ${ }^{12}$ que indicavam que $o$ pico de ulceração ocorria anos após a cirurgia em seres humanos ${ }^{21}$. Entretanto, um trabalho experimental prévio, ${ }^{23}$ que analisou as alterações da mucosa gástrica na gastrojejunostomia após o fechamento pilórico, encontrou úlceras anastomóticas em um período inferior a trinta dias, tanto em anastomoses isoperistálticas como anisoperistálticas, sendo as alterações da mucosa gástrica mais intensas na região da anastomose e no grupo anisoperistáltico. É possível que o refluxo biliopancreático para o estômago seja responsável pelas alterações encontradas na mucosa perianastomótica e que estas não tenham sido influenciadas pela vagotomia.

Podemos concluir com este estudo que, na cerclagem do piloro gastroduodenal de ratos, não houve diferença entre os fios absorvíveis quanto ao tempo de sua reabertura, que ocorreu em um tempo menor ao verificado com o fio inabsorvível. O fio inabsorvível parece ser mais adequado para a manutenção do piloro fechado por um tempo mais prolongado, e não leva à oclusão pilórica permanente. A vagotomia não influencia no tempo de reabertura pilórica, mas acompanha-se de menor reação inflamatória perianastomótica.

\section{AGRADECIMENTOS}

Os autores agradecem à Dra. Ana Paula Coutinho Bello pelo desenho esquemático. À Dra. Ana Cláudia Máximo de Almeida pela tradução do resumo para a língua inglesa. Somos gratos também ao CNPq e à FAPEMIG pelo auxílio finaceiro que possibilitou a realização deste trabalho. 


\begin{abstract}
Objective: The duodenal damage occurs in about $3 \%$ to $12 \%$ of patients with abdominal traumas. The suture of this wound and local drainage is the most common treatment in minor injuries. The challenge remains choosing the best option to treat larger wounds. The closing of the pylorus and the deviation of the digestive transit through a gastrojejunal anastomosis is the most frequent choice in these situations. The objectives of this study were to observe the differences between the time of the pyloric reopening after the occlusion with different suture material and the influence of vagotomy on the local tissue changes. Methods: Thirty rats were studied and submitted to the closure of the gastroduodenal pylorus and gastrojejunal anastomosis. The animals were divided into three groups $(n=10)$, according to the type of suture material: plain catgut, polyglycolic acid and polypropilene. Half of the animals in each group $(n=5)$ were submitted to a troncular vagotomy as well. The studies after the operations consisted of abdominal $x$-ray following intragastric injection of contrast, on a weekly basis until the gastroduodenal transit has been established or during a maximum period of four weeks. At the end of the study, pyloric region and the gastrojejunal anastomosis were removed for histological analysis. The comparison among the groups were performed by the Kaplan-Meier test, with significance for $p<0.05$. Results: Polypropilene suture maintained the pyloric closure during a longer period $(36.3 \pm 11.6$ days) $(p<0.05)$. There were no differences between the polyglicolic acid suture $(25.8 \pm 14.2$ days $)$ and the plain categut suture (18.7 \pm 10.2 days). The vagotomy did not influence the time of the pyloric re-opening, but was associated to less gastric inflammatory reaction. Conclusions:The inabsorbable suture was the most adequate for the exclusion of the pyloric transit and the vagotomy had no influence in the time of the re-opening of the pylorus, but reduced the gastric inflammation.
\end{abstract}

Key words: Duodenum; Pylorus; Vagotomy; Radiology; Rats.

\section{REFERÊNCIAS}

1. Acênsio JA, Feliciano DV, Britt LD, et al. Management of duodenal injuries. Curr Probl Surg.1993;30(11):1023-93.

2. Cave WH. Duodenal injuries. Am J Surg.1946;72(1):26-31.

3. Jorge Filho I, Takahashi PK. Fístulas entéricas pós-operatórias. Rocha PRS, Rodrigues MAG. Complicações em cirurgia do aparelho digestivo. Clínica Brasileira de Cirurgia. Ano IV. Atheneu. 1998. v.II, p.161-80.

4. Morton JR, Jordan GL. Traumatic duodenal injuries. Review of 131 cases. J Trauma.1968;8(2):127-39.

5. Graham JM, Mattox KL, Vaughan GD, et al. Combined pancreatoduodenal injuries. J Trauma.1979;19(5):340-6.

6. Ivatury RR, Nassoura ZE, Simon RJ, et al. Complex duodenal injuries. Surg Clin North Am. 1996;76(4):797-812.

7. Lucas CE. Diagnosis and treatment of pancreatic and duodenal injury. Surg Clin North Am.1977;57(1):49-65.

8. Thal AP, Wilson RF. A pattern of severe blunt trauma to the region of the pancreas.SSurg Gynecol Obstet.1964;119:773-8.

9. Vaughan GD, Frazier OH, Graham DY, et al. The use of pyloric exclusion in the management of severe duodenal injuries. Am J Surg.1977;134(6):785-90.

10. Feliciano DV, Martin TD, Cruse PA, et al. Management of combined pancreatoduodenal injuries. Ann Surg.1987;205(6):67380 .

11. Ivatury RR, Gaudino J, Ascer E, et al. Treatment of penetrating duodenal injuries: primary repair vs. Repair with decompressive enterostomy/serosal patch. J Trauma.1985;25(4):337-41.

12 Donovan AJ, Hagen WE. Traumatic perforation of the duodenum. Am J Surg. 1966;111(3):341-50.

13. Snyder WH, Weigelt JA, Watkins WL, et al. The surgical management of duodenal trauma. Arch Surg.1980;115(4):422-9.

14. Berne CJ, Donovan AJ, White EJ, et al. Duodenal "diverticulization" for duodenal and pancreatic injury. Precepts based on a review of 247 cases. Am J Surg. 1974;127(5):503-7.

15. Lucas CE, Ledgerwood AM. Factors influencing outcome after injury. J Trauma.1975;15(10):839-45.
16. Resende Neto JB. Avaliação da resistência de suturas duodenais com ou sem omentoplastia pediculada [dissertação]. Belo Horizonte (MG): Universidade Federal de Minas Gerais; 1998.

17. Silva AL, Petroianu A. Estudo comparativo entre os controles radiológicos de trânsito gastrintestinal com contraste iodado e baritado, em pacientes submetidos a vagotomia, antrectomia e anastomose gastroduodenal ao nível da curvatura menor, por úlcera cloridropéptica. An Fac Med Univ Fed Minas Gerais. 1985;34(1):13-20.

18. Petroianu A, Silva AL, Carvalho DG, et al. Resultados tardios da vagotomia, antrectomia e anastomose gastroduodenal na curvatura gástrica menor para tratamento de úlcera péptica. Rev Col Bras Cir.1997;24(4):305-9.

19. Sewell WR, Wiland J, Craver BN. New method of comparing sutures of ovine catgut with sutures of bovine catgut in three species. Surg Gynecol Obstet. 1955;100:483-94.

20. De Santis M, Devereux DF, Thompson D. Pyloric exclusion. Suture material choice. Am Surg.1987;53(12):711-4.

21. Martin TD, Feliciano DV, Mattox KL, et al. Severe duodenal injuries. Treatment with pyloric exclusion and gastrojejunostomy. Arch Surg.1983;118(5):631-5.

22. Petroianu A, Silva AL. Estudo da motilidade de segmentos isolados do estômago, antes e após antrectomia e anastomose gastroduodenal ao nível da curvatura menor e em estômagos não operados de cães. AMB Rev Assoc Med Bras.1982;28(5/6): 123-8.

23. Trapiello-Neto V, Santos BMR, Petroianu A, et al. Alterações da mucosa gástrica na gastrojejunostomia isoperistáltica e anisoperistáltica em rato. Arq Gastroenterol.1999;36(2):94-8.

Endereço para correspondência:

Prof.. Andy Petroianu

Avenida Afonso Pena,1.626-apto.1.901

30130-005 - Belo Horizonte, MG.

FONE / FAX: (31) 3274- 7744

E-mail: petroian@medicina.ufmg.br 